SLAC-PUB-8171

June 1999

\title{
Minimum Field Strength in Ultrafast Magnetization Reversal
}

\author{
By C.H. Back et al.
}

Stanford Linear Accelerator Center, Stanford University, Stanford, CA 94309

Work supported by Department of Energy contract DE AC03 76SF00515. 


\title{
Minimum Field Strength in Precessional Magnetization Reversal
}

\author{
C.H. Back ${ }^{1 *}$, R. Allenspach ${ }^{2}$, W. Weber ${ }^{1}$, S.S.P. Parkin ${ }^{3}$, \\ D. Weller ${ }^{3}$, E.L. Garwin ${ }^{4}$, and H.C. Siegmann 1
}

1Laboratorium für Festkörperphysik, ETH Zürich,CH-8093 Zürich, Switzerland. ${ }^{2}$ IBM

Research Division, Zurich Research Laboratory, CH-8803 Rüschlikon, Switzerland. ${ }^{3}$ IBM

Research Division, Almaden Research Center, 650 Harry Rd., San Jose, CA 95120. ${ }^{4}$ Stanford

Linear Accelerator Center, Stanford University, Stanford, CA 94305.

*To whom correspondence should be addressed. E-mail: back@ solid.phys.ethz.ch 
Ultrafast magnetic field pulses as short as 2 ps are able to reverse the magnetization in thin in-plane magnetized cobalt films. The field pulses are applied in the plane of the film and their direction encompasses all angles with the magnetization. At right angle to the magnetization maximum torque is exerted on the spins. In this geometry a precessional magnetization reversal can be triggered by fields as small as $184 \mathrm{kA} / \mathrm{m}$. Applications in future ultrafast magnetic recording schemes can be foreseen. 
Based on experimental advances, magnetization reversal has undergone considerable development in recent years. For instance, it is now possible to observe the direction of the magnetization in nanosized single-domain particles (1-3). In such experiments static magnetic fields are applied. The probability that the magnetization $\vec{M}$ will reverse is determined as a function of the angle at which the external magnetic field $\vec{H}_{e x}$ is applied to the particle. The reversal mechanism is difficult to understand in detail because $\vec{M}$ can assume complex curling and buckling modes depending on the details of the shape and magnetic properties of the particle. A conceptually simpler reversal mode is reversal by precession of the magnetization: no curling and buckling modes occur $(4,5)$. Precessional and conventional reversal differ in the angle between $\vec{M}$ and $\vec{H}_{e x}$ and the duration of the applied field pulse. In conventional magnetic recording, for example, the reversing field is applied antiparallel to the direction of $\vec{M}$, limiting the reversal speed to the nanosecond level $(6,7)$. Much shorter reversal times can be achieved if the external magnetic field inducing the reversal is applied perpendicular to $\vec{M}$ (4). In this case the magnetic field pulse induces a precessional motion of the magnetization vector that leads to magnetization reversal. Precessional reversal in the picosecond regime was demonstrated for thin films magnetized perpendicular to the film plane. However, the magnetic field had to exceed $\approx 2000 \mathrm{kA} / \mathrm{m}$ at a pulse length of a few picoseconds. We demonstrate that considerably smaller field pulses are sufficient to reverse $\vec{M}$ in thin uniaxial in-plane magnetized films. In these thin magnetic sheets the demagnetizing field $\vec{H}_{D}$ helps the externally applied magnetic field $\vec{H}_{e x}$ to induce magnetization reversal. When a short magnetic field pulse provokes the precession of $\vec{M}$ out of the plane of the film, a demagnetizing field $\vec{H}_{D}$ is induced that points normal to the surface of the film. When the external magnetic field pulse is terminated, $\vec{H}_{D}$ persists and the precession of $\vec{M}$ around $\vec{H}_{D}$ completes the magnetization reversal process. In this geometry, magnetization reversal is induced with magnetic field pulses of a few picoseconds' duration, but with small field amplitudes of $<200 \mathrm{kA} / \mathrm{m}$. These amplitudes are well within reach of conventional thin-film recording heads which are capable of producing fields of the order of $400 \mathrm{kA} / \mathrm{m}$. 
Experiments with ultrashort magnetic field pulses require thin ferromagnetic films because the classical skin depth for the penetration of $\vec{H}_{e x}$ into a metal is $\approx 300 \mathrm{~nm}$ for a rise time of 1 ps. The magnetic films used in this study are made of Co with a thickness of $20 \mathrm{~nm}$. Two types of Co films are used, both of which exhibit a uniaxial anisotropy in the plane of the film. One Co film (Co I) was deposited by dc magnetron sputtering on a $\mathrm{MgO}(110)$ substrate. Seed layers of $0.5 \mathrm{~nm} \mathrm{Fe} / 5 \mathrm{~nm} \mathrm{Pt}$ were first deposited at $500{ }^{\circ} \mathrm{C}$ and subsequently the structure $10 \mathrm{~nm} \mathrm{Pt} / 20 \mathrm{~nm} \mathrm{Co} / 2 \mathrm{~nm} \mathrm{Pt}$ was grown at $40{ }^{\circ} \mathrm{C}$. The other Co film (Co II) was electron-beam deposited onto $\mathrm{MgO}(110)$ at $300{ }^{\circ} \mathrm{C}$ as a $30 \mathrm{~nm} \mathrm{Cr} / 20 \mathrm{~nm} \mathrm{Co} / 2 \mathrm{~nm}$ Pt structure. The saturation magnetization for $\mathrm{Co}$ at room temperature is $M_{S}=1360 \mathrm{kA} / \mathrm{m}$. The values of the uniaxial anisotropy field $\vec{H}_{A}$ in the plane of the films, determined using the magneto-optic Kerr effect, are 168 and $160 \mathrm{kA} / \mathrm{m}$ for Co I and Co II, respectively.

The magnetic field pulses were generated using relativistic electron bunches of the Final Focus Test Beam facility at the Stanford Linear Accelerator Center as described previously $(4,5)$. The finely focused electron pulse is capable of producing magnetic fields of several thousand $\mathrm{kA} / \mathrm{m}$ strength and on a micrometer scale. The premagnetized samples were exposed to several pulses at different locations, allowing us to investigate the influence of the pulse length on identical samples. Before each run, the length of the electron pulse was selected and its spatial extent determined at the location of the sample. The number $N$ of electrons per pulse was recorded on a shot-by-shot basis using two toroids in the beam line. The temporal pulse lengths $\sigma_{t}$ were 2,3 , and 4.4 ps.

The electromagnetic fields in the wake of the electron pulse generate considerable destruction in the thin-film samples, but it is limited to distances $R \leq 13 \mu m$ from the center. As shown in previous experiments $(4,5)$, the material remains close to ambient temperature at distances $R \geq 13 \mu \mathrm{m}$. Every point in the $x y$-plane perpendicular to the electron beam receives a magnetic field pulse of the same duration determined by $\sigma_{t}$. For $R \geq 13 \mu \mathrm{m}$, the magnetic field $\vec{H}_{e x}$ is to a good approximation perpendicular to the radius vector $\vec{R}$ and its strength decreases as $1 / \mathrm{R}$. Prior to exposure, the magnetization $\vec{M}$ of the films is set along $-x$, which is the easy magnetization direction. Hence the direction of the magnetic field $\vec{H}_{e x}(x, y)$ encompasses all angles $-\pi \leq \vartheta \leq \pi$ with $\vec{M}$. 
Magnetic information is then obtained in a spin-resolved scanning electron microscope (spin-SEM) (8). The magnetic pattern generated in Co I by a single field pulse of $\sigma_{t}=4.4 p s$ duration (Fig. 1A) shows the initial magnetization direction pointing along $-x$ (white) and the areas that have switched the magnetization direction from $-\vec{M}$ to $+\vec{M}$ (black). The location of impact is at the center of the image, which we also define as the center of the coordinate system. The induced magnetization pattern is symmetric on changing the sign of $x$, but asymmetric on changing the sign of $y$.

We first concentrate on the line $y=0$. On this line, $\vec{H}_{e x} \perp \vec{M}$ and initially the torque $\vec{H}_{e x} \times \vec{M}$ is maximum. The first reversal is $x=110.1 \mu \mathrm{m}$ from the center, corresponding to a magnetic field of $H_{0}=184 \mathrm{kA} / \mathrm{m}$. Towards $x=0$, that means towards larger field values, multiple reversals occur at $x=94.2 \mu \mathrm{m}\left(H_{0}=224 \mathrm{kA} / \mathrm{m}\right), \quad x=79.2 \mu \mathrm{m} \quad\left(H_{0}=264 \mathrm{kA} / \mathrm{m}\right)$, and $x=59.4 \mu \mathrm{m}\left(H_{0}=352 \mathrm{kA} / \mathrm{m}\right)$. On the line with zero average torque $x=0$ no reversal is observed outside the area of beam damage. This shows the fundamental difference between conventional magnetization reversal with $\vec{H}_{e x}$ antiparallel to $\vec{M}$ and precessional reversal. For precessional reversal, the torque $\vec{H}_{e x} \times \vec{M}$ is not equal to zero and is transferred directly from the magnetic sample to the source of the magnetic field, such as the magnetic recording head. No fundamental limit seems to exist for the time interval over which the magnetic field pulse must be applied to induce magnetization reversal, given it has the right amplitude. On the other hand, in conventional magnetization reversal the average torque is equal to zero. In this case, the angular momentum induced by the reversal process must be absorbed by the phonon lattice, a process that is governed by the rate of energy exchange between the lattice and the magnetic system. Thus, the spin lattice relaxation time is the relevant time scale for reversal (9). The multiple reversals along $y=0$ at larger field values hint at a second requirement for precessional reversal. At a given pulse length the magnetic field strength must assume a value in a rather narrow interval, and the product $\sigma_{t} \cdot H_{0}$ becomes important. At this point we also stress the size of the magnetization pattern. Its diameter amounts to $220.1 \mu \mathrm{m}$, about a factor of 5 larger than that observed for the perpendicularly magnetized samples in (4). This indicates that the field strength required for precessional reversal is considerably smaller for in-plane magnetized films, namely only $184 \mathrm{kA} / \mathrm{m}$. 
The magnetic pattern generated in Co II by an identical field pulse (Fig. 1B) shows a similar outer shape to that of Fig. 1A. However, despite having almost equal magnetic anisotropies, the pattern of Co II measures only $136.4 \mu \mathrm{m}$ in diameter. This means that a larger field pulse amplitude is necessary to induce the first reversal, $H_{0}=312 \mathrm{kA} / \mathrm{m}$.

A simple model can explain why the external field needed for reversal is small. Consider a small, single particle that is symmetric in the $x y$-plane with a uniaxial anisotropy field $\vec{H}_{A}$ along the $x$-direction. This particle may have any shape between a sphere and a thin disk. The corresponding demagnetizing field $\vec{H}_{D}$ results from the difference in the demagnetization factor $D_{\|}$for $\vec{M}$ in the $x y$-plane and $D_{\perp}$ for $\vec{M}$ along the $z$-direction, $H_{D}=\left(D_{\perp}-D_{\|}\right) M_{S} / \mu_{0}$. Let us assume coherent rotation of $\vec{M}$ in an external field pulse of Gaussian shape applied along the $y$-direction with an amplitude $H_{0}$ and duration $\sigma_{t}$. If the particle is a sphere, $D_{\perp}-D_{\|}=0$ and hence $H_{D}=0$. This means that for $H_{0}>>H_{A}$, precession of $\vec{M}$ around $\vec{H}_{0}$ takes place. For successful reversal the precession angle $\Phi$ must exceed $\pi / 2$, with $\Phi$ given by the Larmor frequency. This limits the radius for successful reversal to $R_{0}=32 \mu \mathrm{m}$. If we now fill the plane with decoupled spheres we obtain a figure-eight shaped magnetization pattern according to $R(x, y)=R_{0} \sin (\vartheta)$, where $\vartheta$ is the angle between $\vec{M}$ and $\vec{H}_{0}$.

We can now increase $D_{\perp}-D_{\|}$from 0 to 1 , the value for a film. The calculation for the magnetization reversal is performed using the Landau-Lifshitz (LL) equation for each individual particle,

$$
\frac{d \vec{M}}{d t}=-\gamma\left(\vec{M} \times \vec{H}_{t o t}\right)+\frac{\alpha}{M} \vec{M} \times \frac{d \vec{M}}{d t} \mid
$$

The LL equation assumes precession of $\vec{M}$ around the direction of the sum of internal and external magnetic fields $\vec{H}_{t o t}=\vec{H}_{e x}+\vec{H}_{D}+\vec{H}_{A}$, with $\gamma$ being the gyromagnetic ratio $\gamma=0.2212 \cdot 10^{6} \mathrm{~m} / \mathrm{As}$, and relaxation of $\vec{M}$ into the field direction described by the damping constant $\alpha$. We use $\alpha$ as the only parameter to fit the size of the pattern of Fig. 1A. The results for the two different films along $y=0$ (Fig. 3A) show that with increasing demagnetization factor and hence increasing $H_{D}=M_{S} / \mu_{0}$, the size of the pattern grows rapidly. Thus the demagnetizing field plays a crucial role in the reversal process. This leads to the following three-step model for ultrafast reversal. (1) During the field pulse, $\vec{M}$ 
precesses around $\vec{H}_{e x}$ out of the plane of the film. As $\vec{M}$ leaves the plane of the film the effective demagnetizing field increases with the angle $\Theta$ between $\vec{M}$ and the film plane: $H_{D}=\left(M_{S} / \mu_{0}\right) \sin \Theta$. (2) When $\vec{H}_{e x}$ ceases to exist, $\vec{M}$ continues to precess, but now around $\vec{H}_{D}+\vec{H}_{A}$. The maximum angle $\Theta$ assumed by $\vec{M}$ decides whether the magnetization reverses and whether even multiple reversals can occur. (3) Eventually $\vec{M}$ relaxes into one of the two easy magnetization directions. This final step can take up to $500 \mathrm{ps.}$

The size of the pattern for Co II is not reproduced in Fig. 3A. As both Co films have the same magnetization and demagnetization factors and similar uniaxial anisotropy, the decrease in size must be attributed to an increase in the intrinsic damping constant $\alpha(10)$. Plotting the calculated radius as a function of $\alpha$ for Co II with $H_{A}=160 \mathrm{kA} / \mathrm{m}$ (Fig. 3B) agreement with the experiment is reached for $\alpha=0.22$. In the calculated magnetization patterns for Co I and Co II (Figs. 2A B) the difference between these two patterns is due to the different damping constants ( $\alpha=0.037$ for Co I and $\alpha=0.22$ for Co II). We see that the size and the overall outer shape of the patterns are well reproduced by the calculation. The asymmetry of the pattern when $y$ is inverted is caused by the direction of the premagnetization. A further test for the correct choice of $\alpha$ is the comparison of the experimental location of the first reversal with the calculated ones when the duration of the field pulse is varied (Fig. 4). Good agreement is found for the chosen parameters.

Whereas the size and overall shape of the pattern are well explained by the calculation, the inner structure is not. The calculated pattern (Fig. 1A) shows a multitude of rings near the center, whereas experimentally the innermost part is homogeneously magnetized along the original direction. We attribute this discrepancy to the magnetostatic energy at charged domain walls: "zig-zag" boundaries separate regions with opposite $\vec{M}$ (see inset in Fig. 1). The amplitude of the zig-zag is largest when the boundary is perpendicular to the $x$-axis. This is known as the head-on configuration of $\vec{M}$ and has been extensively studied. It occurs in single-crystalline samples and is caused by the large magnetostatic energy of the head-on configuration. In contrast a regular $180^{\circ}$ wall is formed at the boundary running parallel to the $x$-axis. Note that the zig-zag boundary is not reproduced by the calculation because the longrange dipolar interaction between different cells is neglected. The calculated pattern in 
Fig. 2A shows that the reversed (black) part becomes increasingly thinner as the center is approached. At some point, the white areas will overlap due to the zig-zag boundaries. The overlapping leads to annihilation of the thinner black reversed areas. Such annihilation might then explain why only two black reversed areas are observed. The inset in Fig. 1B shows a gradual transition, indicating a structure dominated by grains. Owing to the reduced crystallinity of Co II, zig-zag walls are not formed. Correspondingly, a stable reversed area is seen much closer to the center of the structure.

We note that the radii of the experimentally observed inner rings are not exactly reproduced by the calculations. For example in Co I the second reversal back to the original direction and, even more so, the subsequent third and fourth reversals occur at smaller field values than expected. The same phenomenon is observed in Co II. The details of the inner structure of the patterns cannot be calculated with the simple LL approach unless one assumes that some of the material properties vary with time. Supposing that intrinsic properties such as $M_{S}$ and $H_{A}$ are constant, one is forced to assume that $\alpha$ is time-dependent. Two different mechanisms might lead to a time-dependent effective damping constant $\alpha_{\text {eff }}$. The first one is the excitation of magnons. The field pulses are built with frequencies close to the frequency band of magnons, hence magnon excitation might be enhanced. This can lead to an increase in energy dissipation and thus to an increase in $\alpha_{\text {eff }}$. The other mechanism is electronelectron scattering (11). If $\vec{M}$ precesses at a different rate in each location, this scattering will be very strong, again leading to a larger effective damping constant.

Ultimately, direct observation of $\vec{M}$ during the precessional motion is desired. Freeman and coworkers (12) have shown that this is indeed possible with the magneto-optic Kerr effect using picosecond laser pulses. Other groups employ inductive probing (13) or spin-polarized tunneling (14). Another exciting prospect comes from the development of the Next Linear Collider $(15,16)$. In this project, microstructured electron pulses will be developed that deliver a train of very strong magnetic field pulses of picosecond length, ideal for observing the dynamics of the reversal.

These results have implications for longitudinal magnetic recording and demonstrate the possibility of extremely high-data-rate recording if problems arising from transitions 
C.H. Back et al., page9

between regions of oppositely magnetized material can be overcome. This requires either a magnetic medium consisting of identical grains or single-domain particles (17). 


\section{References}

1. M. Ledermann, S. Schultz, and M. Ozaki, Phys. Rev. Lett. 73, 1986 (1994).

2. W. Wernsdorfer et al., Phys. Rev. Lett. 78, 1791 (1997).

3. S.Y. Chou, P.R. Krauss, and L. Kong, J. Appl. Phys. 79, 6101 (1996).

4. C.H. Back et al., Phys. Rev. Lett. 81, 3251 (1998).

5. H.C. Siegmann et al., J. Magn. Magn. Mat. 151, L8 (1995).

6. A. Lyberatos and R.W. Chantrell, J. Phys. Condens. Matter 9, 2623 (1997).

7. W.D. Doyle, S. Stinnet, C. Dawson, and L. He, J. Magn. Soc. Jpn. 22, 91 (1998).

8. $\quad$ R. Allenspach, J. Magn. Magn. Mat. 129, 160 (1994).

9. Values for the spin lattice relaxation time are found to lie in the realm of $100 \mathrm{ps}$. A. Vaterlaus, T. Beutler, D. Guarisco, M. Lutz, F. Meier, Phys. Rev. B 46, 5280 (1992); A. Scholl, L. Baumgarten, R. Jacquemin, W. Eberhard, Phys. Rev. Lett. 79, 5146 (1997) and references therein.

10. $\alpha$ is in fact very sensitive to the quality of the sample. It is known that only highquality samples show small damping constants. Note also that the value of $\alpha$ might be different from values determined in FMR experiments. In FMR a small, stationary rf excitation causes coherent precession around the effective field. In our experiments, in contrast, the excitation is strong and nonstationary.

11. D. Oberli, R. Burgermeister, S. Riesen, W. Weber, H.C. Siegmann, Phys. Rev. Lett. 81, 4228 (1998).

12. W.K. Hiebert, A. Stankiewicz, and M.R. Freeman, Phys. Rev. Lett. 79, 1134 (1998).

13. T.J. Silva, T.M. Crawford, and C.T. Rogers, in Proceedings of the Joint MMMIntermag Conference, San Francisco, CA, 1998.

14. R. H. Koch et al., Phys. Rev. Lett. 81, 4512 (1998).

15. T. Nakanishi, in Proceedings of LE '98, St. Petersburg, Sept. 2-8, 1998.

16. C. Adolphson et al., "Zeroth-Order Design Report for the Next Linear Collider", SLAC Report 474 (1996).

17. C. Stamm et al., Science 282, 449 (1998). 
18. We gratefully acknowledge the valuable input of several people at the FFTB and PEL, and the assistance of D. Guarisco with some of the programming. C.H. Back gratefully acknowledges the Swiss National Fund for financial support. This work was supported in part by the Department of Energy under contract No. DE-AC03$76 \mathrm{SF} 00515$. 


\section{Figures}

Fig. 1 Magnetization pattern written into uniaxial cobalt films with a single electron pulse of $\sigma_{t}=4.4 \mathrm{ps}$ duration. The images were measured with spin-SEM. The samples were premagnetized along the $-x$ direction. In the white areas the magnetization points along the $-x$ direction, in the black areas it has been reversed to the $+x$ direction. (A) Image of Co I. The inset shows zig-zag domain walls separating areas of opposite $\vec{M}$. The contour lines of the pattern approximately represent lines of constant angular momentum transferred by the field pulse. (B) Image of Co II. The inset shows a gradual transition between areas of opposite $\vec{M}$.

Fig. 2 Magnetization patterns calculated for the two different films using the LandauLifshitz equation with $M_{S}=1360 \mathrm{kA} / \mathrm{m}$. (A) The parameters for Co I are $H_{A}=168 \mathrm{kA} / \mathrm{m}$ and $\alpha=0.037$, (B) while for Co II we use $H_{A}=160 \mathrm{kA} / \mathrm{m}$ and $\alpha=0.22$. The difference to (A) is caused by the increase of $\alpha$.

Fig. 3 (A) Calculated distance of the first reversal along $y=0$ for $\sigma_{t}=4.4 p s$ and $M_{S}=1360 \mathrm{kA} / \mathrm{m}$ versus the difference $D_{\perp}-D_{\|}$of the demagnetization factors for $\vec{M}$ perpendicular and parallel to the plane of the film for a fixed $\alpha$ of 0.037 . (B) Calculated distance versus the damping constant $\alpha$ for $D_{\perp}-D_{\|}=1$.

Fig. 4 Measured and calculated values of the position of the first reversal along $y=0$ versus the length of the magnetic field pulse $\sigma_{t}$. The upper curve represents Co I, the lower Co II. For the calculations we assume $M_{S}=1360 \mathrm{kA} / \mathrm{m}$ and $D_{\perp}-D_{\|}=1$. For Co I we use $H_{A}=168 \mathrm{kA} / \mathrm{m}$ and $\alpha=0.037$, for Co II $H_{A}=160 \mathrm{kA} / \mathrm{m}$, and $\alpha=0.22$. The number of electrons per pulse is held constant in these experiments at $(9.1 \pm 0.2) \cdot 10^{9}$. 

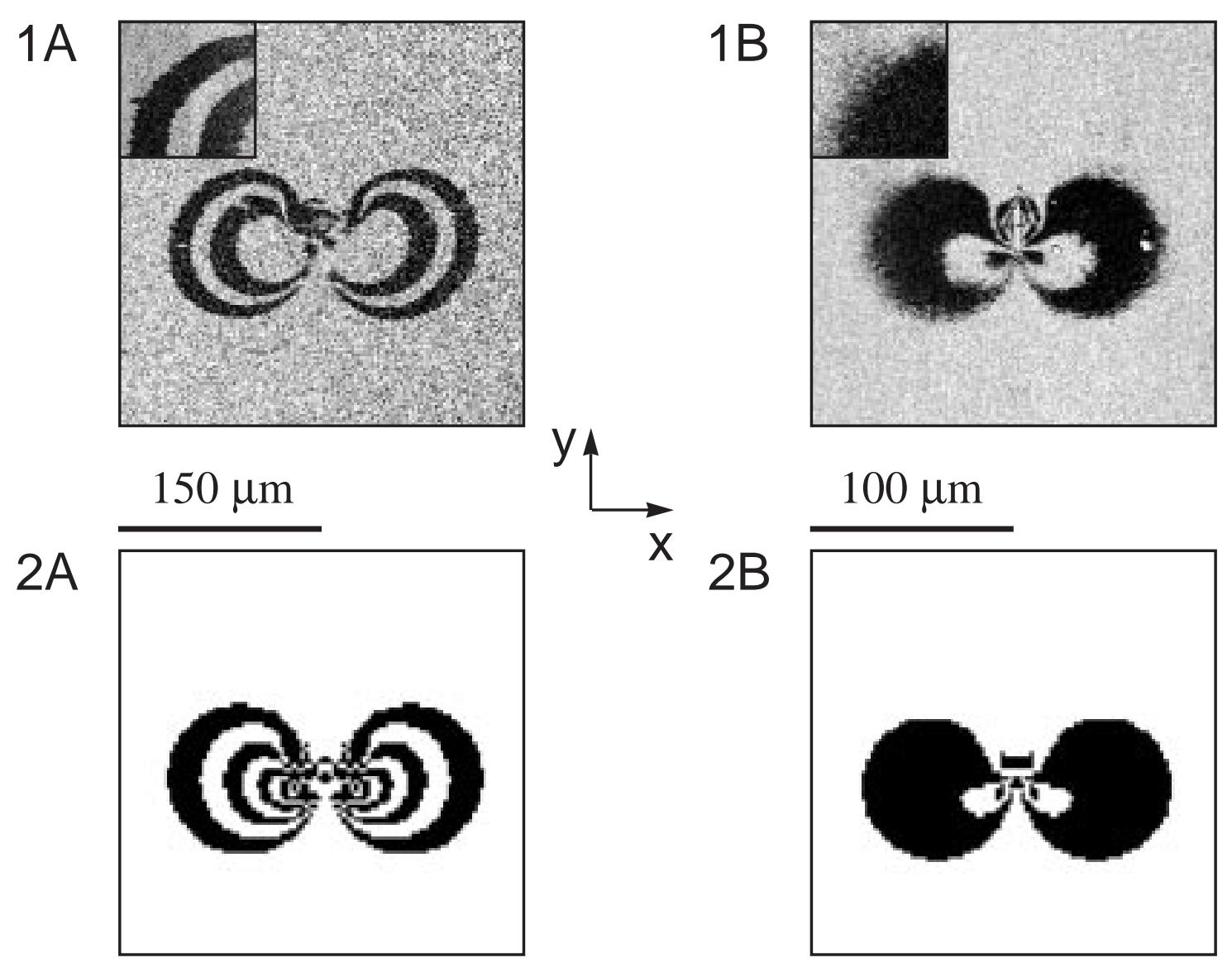

Figure 1

Figure 2 

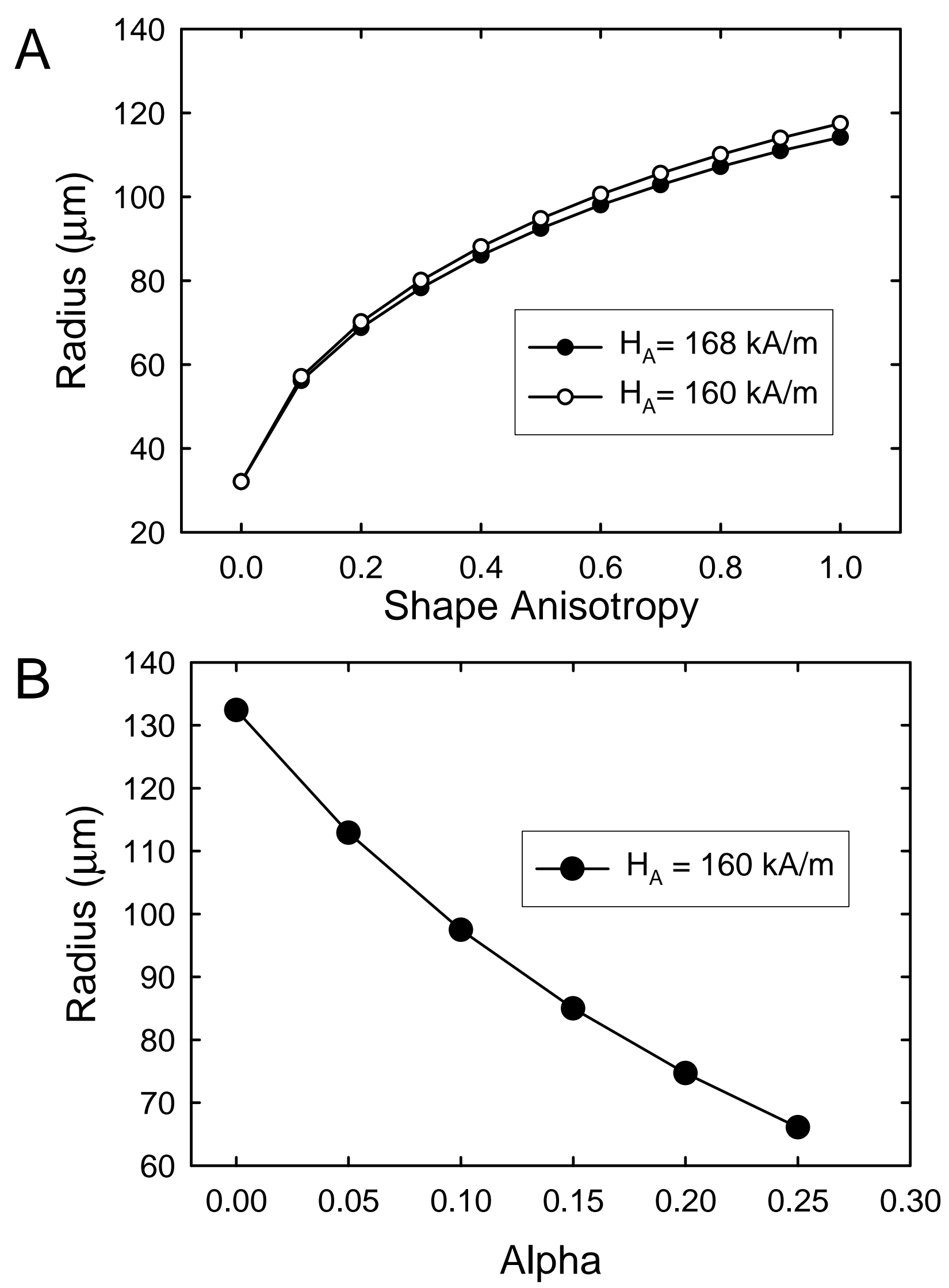

Figure 3 


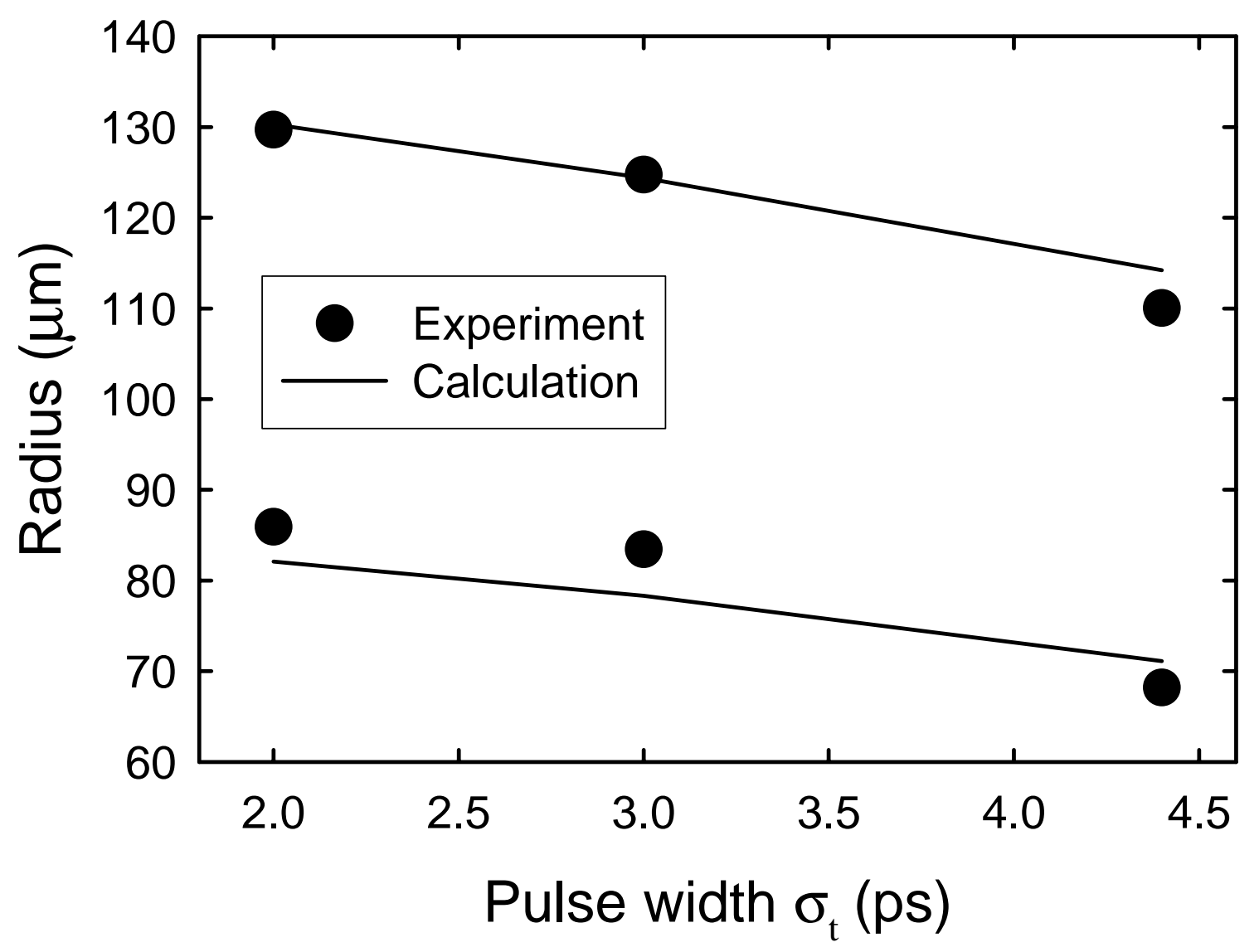

Figure 4 